\title{
Análise da fluência por compressão no isolamento ao ruído de impacto de placas cimentícias com resíduo de EVA
}

\author{
Analysis of creep under compression on impact noise \\ insulation of cementitious plates with EVA waste
}

\section{Fabianne Azevedo dos Santos \\ Aluísio Braz de Melo \\ Roberto Leal Pimentel \\ Maria Fernanda de Oliveira}

\section{Resumo}

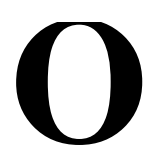

s ruídos de impacto nas edificações multipavimentos são reduzidos com a execução de piso flutuante, caracterizado pela inclusão de um material resiliente entre o contrapiso e a laje. Nesse estudo, buscou-se avaliar a influência da compressão desse material no desempenho acústico do sistema. Dois materiais resilientes foram comparados, um produzido em laboratório (placas cimentícias com resíduos EVA - PEVA1,8), e outro comercial (manta recicladas de resíduos PET - MantaPET). As medições visando relacionar a rigidez dinâmica dos materiais e o desempenho acústico ocorreram em três anos. Adicionalmente, foram feitas estimativas de comportamento para dez anos, baseando-se em modelos teóricos e teste de fluência à compressão. Enquanto a PEVA1,8 apresentou redução em sua rigidez dinâmica $(20,0 \mathrm{MN} / \mathrm{m}$ para $17,7 \mathrm{MN} / \mathrm{m}$ ) ao longo do tempo, a MantaPET teve aumento nesse parâmetro (2,0 MN/m para 3,5 MN/m ), porém ambas mantiveram o desempenho intermediário (conforme NBR 15575-3:2013) ao ruído de impacto, com L ${ }_{\mathrm{nT}, \mathrm{w}}$ igual a $60 \mathrm{~dB}$ e $56 \mathrm{~dB}$ em um piso de testes respectivamente. As estimativas para dez anos indicaram deformação menor para PEVA1,8 (3,42\%) comparativamente à MantaPET $(20,15 \%)$ e confirmaram a tendência de variação distinta na rigidez dinâmica, sem comprometimento do desempenho acústico desses materiais.

Palavras-chave: Material reliente. Fluência. Ruído de impacto. Rigidez dinâmica.

${ }^{1}$ Fabianne Azevedo dos Santos ${ }^{1}$ Universidade Federal da Paraíba João Pessoa - PB - Brasil

${ }^{2}$ Aluísio Braz de Melo 2Universidade Federal da Paraíba João Pessoa - PB - Brasil

${ }^{3}$ Roberto Leal Pimentel ${ }^{3}$ Universidade Federal da Paraíba João Pessoa - PB - Brasil

${ }^{4}$ Maria Fernanda de Oliveira ${ }^{4}$ Universidade do Vale dos Sinos São Leopoldo - RS - Brasil

Recebido em 09/08/20 Aceito em 08/08/21

\section{Abstract}

Impact noise in multi-floor buildings is reduced with the execution of a floating floor, which has a resilient material between the subfloor and the slab. In this study, the evaluation of the influence of compression of this material on the acoustic performance of the system was sought. Two resilient materials were compared, one being produced in the laboratory (cementitious plates with EVA - PEVA1,8 waste), and the other commercially (blanket recycled from PET waste-MantaPET). The measurements aiming to relate the dynamic stiffness of the materials and the acoustic performance were up to three years. Additionally, estimates were made for ten years, based on theoretical models and creep compression tests. While PEVAl,8 decreased its dynamic stiffness $\left(20.0 \mathrm{MN} / \mathrm{m}^{3}\right.$ to $\left.17.7 \mathrm{MN} / \mathrm{m}^{3}\right)$ over time, MantaPET increased $\left(2.0 \mathrm{MN} / \mathrm{m}^{3}\right.$ to $\left.3.5 \mathrm{MN} / \mathrm{m}^{3}\right)$, but both maintained intermediate performance (according to NBR 15575-3:2013) to impact noise, presenting $L{ }_{n T, w}$ equal to $60 \mathrm{~dB}$ and $56 \mathrm{~dB}$ in a test floor, respectively. Estimates for ten years indicated less deformation for PEVA1,8 (3.42\%), compared to MantaPET (20.15\%) and confirmed the trend of changes in the dynamic stiffness, without compromising the acoustic performance of these materials.

Keywords: Resilient material. Creep. Impact noise. Dynamic stiffness.

SANTOS, F. A. dos; MELO, A. B. de; PIMENTEL, R. L.; OLIVEIRA, M. F. de. Análise da fluência por compressão no isolamento ao ruído de impacto de placas cimentícias com resíduo de EVA. Ambiente Construído, Porto Alegre, v. 22, n. 1, p. 125-138, jan./mar. 2022. 


\section{Introdução}

Na concepção das construções é possível fazer uso de novos materiais alternativos que possam substituir alguns materiais convencionais, sem finalidade estrutural. Essa prática possibilita uma solução para destinação de resíduos e uma tentativa de melhora no desempenho do ambiente construído.

Um dos problemas em habitações multifamiliares verticais, quando projetadas sem tratamento específico para isolamento acústico, é o ruído de impacto entre pisos. Esse ruído se propaga pela laje, alcança as paredes e repercute nas unidades habitacionais vizinhas, o que gera desconfortos auditivos para os moradores.

O isolamento acústico nas edificações é tratado na NBR 15575-3 (ABNT, 2013), na qual há valores que classificam os sistemas de piso que separam as unidades habitacionais autônomas posicionadas em pavimentos distintos e que precisam atingir pelo menos o nível mínimo de $80 \mathrm{~dB}$ de nível de pressão sonora de impacto ponderado ( $\left.\mathrm{L}_{\mathrm{nT}, \mathrm{w}}^{\prime}\right)$.

Souza, Almeida e Bragan (2006) explicam que a forma de tratamento para atenuar ruídos varia com o tipo (contínuo, intermitente, impacto, etc.), as fontes e sua distribuição (pontuais, lineares e planas), e com os modos de propagação (aérea, estrutural). A forma de atenuação dos ruídos aéreos depende da posição da fonte emissora e da do receptor.

Para mitigar o problema de propagação do ruído de impacto é comumente usado o sistema de piso flutuante, o qual consiste em um conjunto formado pelo contrapiso revestido, apoiado sobre um material resiliente, que separa o contrapiso da laje estrutural e das paredes do edifício e que cumpre o papel de mitigador da energia sonora. O desempenho do sistema vai depender de alguns fatores, como tipo de revestimento do piso, tamanho do ambiente, tipologia da laje e rigidez dinâmica do material resiliente.

Uma alternativa é o uso do resíduo de EVA em componentes na construção civil, o que ajudaria a reduzir os impactos ambientais causados pelos volumes descartados pelas indústrias calçadistas. Alternativamente ao destino mais comum para tais resíduos, que é alimentar fornos de indústrias cimentícias, pode-se oferecer uma opção interessante ao explorar as propriedades do material para melhorar a qualidade do ambiente construído, nesse caso específico o isolamento acústico entre unidades autônomas de edifícios com vários pavimentos, analisando o comportamento desse material em relação ao tempo de uso após ser aplicado em pisos flutuantes.

A problemática em foco no presente trabalho trata da possibilidade de alteração da rigidez dinâmica do material resiliente empregado, em longo prazo, após ser instalado no sistema de piso flutuante, bem como na possível variação de seu desempenho relativamente ao isolamento acústico.

Nesse contexto, o objetivo deste trabalho é estudar a influência da compressão de material resiliente, neste caso placas cimentícias com agregados leves de EVA instaladas entre o contrapiso e a laje, ao longo de sua vida útil, visando estabelecer em que medida há comprometimento na capacidade de redução dos ruídos de impacto no sistema de piso flutuante.

\section{Referencial teórico}

De acordo com a NBR 15575-3 (ABNT, 2013), o nível de pressão sonora de impacto ponderado ( $\left.{ }^{\prime}{ }_{n T, w}\right)$ de cada sistema é o que vai classificar o desempenho da edificação. Se o $L^{\prime}{ }_{n T, w}$ for entre $66 \mathrm{~dB}$ e $80 \mathrm{~dB}$, o sistema do piso é considerado com nível de desempenho mínimo (M); para valores entre $56 \mathrm{~dB}$ e $65 \mathrm{~dB}$, classifica-se com nível de desempenho intermediário (I); e para valores menores ou iguais a $55 \mathrm{~dB}$, classifica-se com nível de desempenho superior (S).

Um fator que pode influenciar os valores do desempenho acima é o tipo de laje estrutural. A laje maciça, diferentemente dos outros tipos, apresenta uma solidez que potencialmente possibilita um isolamento apropriado quanto ao ruído de impacto. Segundo a Câmara Brasileira para a Indústria da Construção (CÂMARA..., 2013), lajes de concreto armado de $10 \mathrm{~cm}$ a $12 \mathrm{~cm}$ de espessura conseguem potencialmente atender ao nível mínimo da NBR 15575-3 (ABNT, 2013), mesmo sem se utilizar um sistema de piso flutuante.

Nunes, Zini e Pagnussat (2014) dizem que sistemas heterogêneos diferentes da laje maciça, como laje prémoldada, têm diversos benefícios estruturais e econômicos, mas podem apresentar fragilidades no isolamento. Porém, as deficiências decorrentes da reduzida espessura e da ausência de vínculo entre as peças 
desses sistemas heterogêneos podem ser solucionadas com a utilização de materiais leves e de base cimentícia, em camadas alternadas.

Segundo Bistafa (2011), o piso flutuante é mais eficaz se estiver desacoplado das paredes, ou seja, o material resiliente deve ser inserido também nas ligações verticais entre paredes e contrapiso, idealmente isolando-se o contrapiso da parede e de toda a estrutura. Esse isolamento lateral é importante para evitar o aparecimento de pontes acústicas nas ligações da estrutura como, por exemplo, a ligação do piso com a parede feita pelo rodapé (FERRAZ, 2008).

\section{Rigidez dinâmica}

A rigidez dinâmica do material resiliente em um sistema de piso flutuante é uma propriedade importante para propiciar a redução dos ruídos de impacto nesse sistema. A Figura 1 apresenta uma analogia mecânica do sistema de piso flutuante, através de um sistema massa-mola, em que $m$ é a massa por unidade de superfície do piso flutuante, $k$ é a rigidez dinâmica por unidade de superfície do material resiliente e $c$ é o amortecimento por unidade da superfície desse mesmo material.

Os ensaios para a determinação da rigidez dinâmica $k$ são realizados com base nos procedimentos da norma ISO 9052-1 (INTERNATIONAL..., 1989). A determinação da rigidez dinâmica aparente $\left(s^{\prime} t\right)$, por unidade de área da amostra, pode ser feita pelo método da ressonância, que consiste na medição da frequência fundamental de ressonância da vibração vertical do sistema massa-mola. A mola corresponde ao material resiliente; a massa, à placa de carga posta sobre o material a ser ensaiado; e a frequência de ressonância $\left(f_{r}\right)$ da amostra ensaiada, em Hz, é dada pela Equação 1.

$f_{r}=\frac{1}{2 \pi} \sqrt{\frac{s_{t}^{\prime}}{m_{t}^{\prime}}}$

Eq. 1

Sendo:

$s ' t$ a rigidez dinâmica aparente por unidade de área da amostra $(\mathrm{N} / \mathrm{m})$; e

m't a massa total por unidade de área usada durante o teste $(\mathrm{kg} / \mathrm{m})$.

O cálculo da rigidez dinâmica aparente por unidade de área da amostra em análise, $s$ 't, é dada pela Equação 2, obtida diretamente da Equação 1.

$s_{t}^{\prime}=4 \pi^{2} m_{t}^{\prime} f_{r}^{2}$

Eq. 2

\section{Fluência à compressão}

Um fenômeno adicional é o da alteração na rigidez dinâmica de um material resiliente em um sistema de piso flutuante, tendo em conta a carga compressiva da camada de material (contrapiso) sobre ele. Borges et al. (2018) afirmam que o que pode determinar a eficiência do isolamento de ruído de impacto é a capacidade de deformação do material resiliente submetido à carga constante. Para isso, torna-se importante executar teste de compressão de amostras que traduza uma relação entre os fatores de tempo de ensaio, deformação e tensão aplicada. Portanto, tais resultados permitirão avaliar o desempenho do sistema ao longo do tempo, em função das taxas de carregamento durante o uso de dado sistema de piso flutuante.

Figura 1 - Analogia mecânica do sistema de piso flutuante

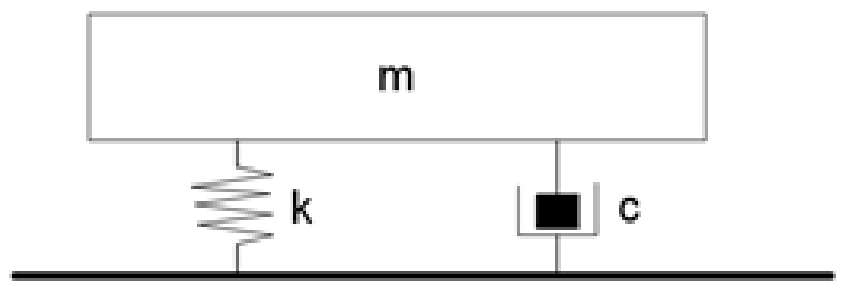


A fluência por compressão é uma propriedade que pode ser determinada com base no carregamento do material resiliente ao longo do tempo, de acordo com os procedimentos da ISO 20392 (INTERNATIONAL..., 2007). Nesse caso, a espessura do material resiliente sob carga é medida por 90 dias, e as medições são utilizadas para estimar a espessura do material após 10 anos de uso em dado piso flutuante. A deformação do material resiliente no longo prazo é calculada com base na Equação de Findley (Equação 3), disponível na ISO 20392 (INTERNATIONAL..., 2007).

$X_{t}=x_{0}+m t^{b}$

Sendo:

$X_{t}$ : encurtamento, em milímetros, no tempo $t$ (em horas);

$x_{0}$ : encurtamento inicial (60 s após o carregamento); e

$m, b$ : constantes dependentes do material em questão, calculadas por análise de regressão da deformação medida.

Posteriormente, é calculada a deformação relativa da amostra, $\varepsilon_{t}$, em porcentagem, para 10 anos de uso, usando a Equação 4.

$\varepsilon_{t}=\left(X_{t} / d_{s}\right) \times 100$

Sendo $d_{s}$ : espessura inicial, em milímetros.

O estudo de Klippel Filho et al. (2017) é um exemplo de resultado de estimativa de isolamento ao ruído de impacto com o uso da lã de PET com $10 \mathrm{~mm}$ de espessura. Os autores identificaram que a deformação estimada após 10 anos de uso dos materiais sob carga mostrou variações de $11 \%$ a 39\%. As amostras com maior densidade $(0,24 \mathrm{~kg} / \mathrm{m}$ e $0,32 \mathrm{~kg} / \mathrm{m})$, como esperado, mostraram deformações menores, enquanto nas amostras de menor densidade $(0,18 \mathrm{~kg} / \mathrm{m}$ e $0,22 \mathrm{~kg} / \mathrm{m})$ as deformações foram maiores. Nesse caso, pode-se observar que a deformação depende da densidade, mesmo que as composições tenham a mesma espessura.

\section{Estimativa de isolamento ao ruído de impacto}

De acordo com a ISO 12354-2 (INTERNATIONAL..., 2017), é possível se prever o comportamento quanto ao isolamento de ruídos de impacto de um material resiliente usado em um piso flutuante sem a necessidade do ensaio de ruído de impacto. O valor do isolamento previsto é obtido através do cálculo da redução do nível de pressão sonora de ruído de impacto $(\Delta \mathrm{L}$, em $\mathrm{dB})$ em relação a um piso sem o material resiliente (Equação 5), utilizando o valor da rigidez dinâmica, após o ensaio de fluência à compressão desse material. Ou seja, essa é uma possibilidade para acessar informações sobre a eventual alteração da rigidez dinâmica do material resiliente ao longo do tempo de sua vida útil, após ser instalado no sistema de piso flutuante, bem como se há relação com a variação de seu desempenho ao isolamento de ruído de impacto.

$\Delta \mathrm{L}=\left(30 \log \frac{f}{f_{o}}\right)$

Eq. 5

Sendo:

$f$ : frequência central de banda de oitava, adotada como $500 \mathrm{~Hz}$; e

$f_{o}$ : frequência de ressonância do sistema em Hz, obtida a partir da Equação 1.

Entretanto, $f_{o}$ é agora calculada utilizando a massa superficial do sistema de piso flutuante (contrapiso mais revestimento), ao passo que $f_{r}$ (vide Eq. 1) é calculada utilizando a massa superficial colocada sobre a amostra testada. A frequência central de banda de oitava $(f)$ foi adotada como $500 \mathrm{~Hz}$, de acordo com Schiavi et al. (2007).

Borges (2015), ao estudar o resíduo de EVA utilizado em placas cimentícias para isolamento ao ruído de impacto, verificou que as estimativas de deformação das amostras de EVA, com $3 \mathrm{~cm}$ e $5 \mathrm{~cm}$ de espessura, apresentaram correlação com o aumento na proporção de resíduos, sendo previstas por extrapolação de valores deformações maiores nas amostras confeccionadas com 50\% e 75\% de EVA. Os resultados demonstraram que os compósitos com mais adição de resíduos apresentaram valores de redução dos sons de impacto $(\Delta \mathrm{L})$ superiores, com redução de $22 \mathrm{~dB}$ para EVA $50 \%$ e de $24 \mathrm{~dB}$ para EVA $75 \%$ em relação ao sistema de referência sem EVA.

No estudo de Klippel Filho et al. (2017), as diferenças nos valores de rigidez dinâmica após o ensaio de fluência à compressão indicaram perdas inferiores no isolamento acústico ao ruído de impacto de $1 \mathrm{~dB} \mathrm{em}$ todas as amostras de material resiliente de resíduo de PET com $10 \mathrm{~mm}$ de espessura, só se diferenciando a

128 Santos, F. A. dos; Melo, A. B. de; Pimentel, R. L.; Oliveira, M. F. de 
densidade entre elas $(0,18 \mathrm{~kg} / \mathrm{m}, 0,22 \mathrm{~kg} / \mathrm{m}, 0,24 \mathrm{~kg} / \mathrm{m}$ e $0,32 \mathrm{~kg} / \mathrm{m})$. Na amostra com maior densidade a redução estimada na capacidade de isolamento ao ruído de impacto foi baixa, com $0,2 \mathrm{~dB}$. O estudo concluiu que, após 10 anos de uso sob uma carga de $200 \mathrm{~kg} / \mathrm{m} \mathrm{em} \mathrm{um} \mathrm{sistema} \mathrm{de} \mathrm{piso} \mathrm{flutuante,} \mathrm{espera-se} \mathrm{perda} \mathrm{na}$ eficiência no isolamento ao ruído de impacto de, no máximo, $1 \mathrm{~dB}$ nas amostras testadas.

\section{Resíduo de EVA}

EVA é um copolímero utilizado na indústria calçadista para a confecção de solados e palmilhas internas de calçados. A geração do resíduo está relacionada ao processo de corte das mantas de EVA (aparas) na produção de solas, entressolas e palmilhas dos calçados e no lixamento (pó) na fase de acabamento dos calçados. Esses resíduos vêm sendo estudados em aplicação em sistema de piso flutuante.

De acordo com Brancher et al. (2016), materiais de construção com aplicação não estrutural, que não requerem elevada resistência mecânica, podem conter elevadas porcentagens dos resíduos de EVA. Outra potencial aplicação é aproveitar os resíduos de EVA para aumentar a eficiência no isolamento térmico e acústico de elementos construtivos.

De acordo com Garlet (1998), foi observado que esse material tem características relevantes para fins de reciclagem mecânica e tem propriedades para ser um material resiliente, que pode contribuir para reduzir ruídos de impacto em sistemas de piso flutuante.

Segundo Kim et al. (2015), na Coreia do Sul os materiais resilientes mais comuns utilizados em pisos flutuantes são poliestireno expandido (EPS) e EVA. Estudos experimentais como os de Kim et al. (2015), Brancher et al. (2016), Tutikian et al. (2017) e Borges et al. (2018) têm demonstrado perspectivas positivas quanto à utilização do resíduo do EVA para reduzir ruído de impacto entre pisos de edificações multipavimentos.

Tutikian et al. (2013) avaliaram o desempenho acústico de compósitos cimentícios com resíduos de EVA para reduzir ruídos de impactos em pisos. Utilizaram para a fabricação das placas cimentícias com EVA $(50 \mathrm{~cm} \times 50 \mathrm{~cm} \times 3 \mathrm{~cm}$ ) as dosagens de $80 \%, 70 \%$ e $60 \%$ de agregado graúdo de EVA. Os resultados indicaram que as amostras com $80 \%$ e $60 \%$ desse agregado apresentaram número global (L' ${ }_{n T, w}$ ) de $56 \mathrm{~dB}$ e de $62 \mathrm{~dB}$ respectivamente, mostrando uma melhora de $6 \mathrm{~dB}$ no isolamento acústico, na medida em que se aumentou o teor de EVA em $20 \%$.

\section{Método}

A análise de longo prazo foi estabelecida como o principal aspecto a ser considerado no presente trabalho para se avaliar o comportamento de materiais resilientes quanto a sua aplicação em sistemas de piso flutuante. A placa cimentícia com EVA proposta foi comparada com uma manta composta de fibras recicladas de resíduo de PET (polímero de tereftalato de polietileno) utilizada comercialmente como material resiliente em pisos flutuantes. Essa manta tem $8 \mathrm{~mm}$ de espessura e $30 \mathrm{~kg} / \mathrm{m}$ de densidade, e foi intitulada no presente trabalho como MantaPET. Salienta-se que esses materiais comparados não têm composição similar, e os resultados obtidos serviram somente para se ter uma ideia da diferença de comportamento do desempenho da placa cimentícia proposta em relação a um material que já está inserido no mercado e que também tem como essência e utilização de resíduos. $O$ foco da análise foi o comportamento em função da influência da compressão ao longo do tempo.

As placas cimentícias foram denominadas pela abreviação PEVA1,8, sendo o numeral 1,8 relacionado à espessura delas $(1,8 \mathrm{~cm})$ e com três lotes diferentes (PEVA1,8_B, PEVA1,8_C e PEVA1,8_D) para se ter a possibilidade de variar o tempo de medição entre essas amostras e, com isso, obter maior variedade de resultados. A MantaPET também teve lotes diferentes para medição, designados por MantaPET_A e MantaPET_B.

A placa PEVA1,8 (Figura 2) foi fabricada com resíduo de EVA (agregado leve com diâmetro máximo característico de $6,3 \mathrm{~mm}$ e massa unitária de $90,28 \mathrm{~kg} / \mathrm{m}$ ) e cimento CP II-F 32, na dosagem do traço em massa de 1:8 (cimento: EVA) com relação água/cimento igual a $0,50 \mathrm{~cm}$ e a $1,8 \mathrm{~cm}$ de espessura.

A escolha do cimento utilizado foi devido a sua abrangência de utilização em estruturas em concreto armado, e argamassas de assentamento e revestimento. O material, depois de homogeneizado, foi depositado em forma metálica com tampa, sendo o conjunto submetido à prensagem com carga de $6.000 \mathrm{~N}$. As placas PEVA1,8 foram desmoldadas após $24 \mathrm{~h}$ e levadas a uma estufa a $100{ }^{\circ} \mathrm{C}$ por igual período, o que 
proporcionou a desejável redução da densidade da amostra $(388,90 \mathrm{~kg} / \mathrm{m})$ para que a placa ficasse mais leve.

Para avaliar o potencial de isolamento dos materiais resilientes comparados, as amostras foram submetidas a ensaios de ruído de impacto (RI) (INTERNATIONAL..., 2015), rigidez dinâmica (RD) (INTERNATIONAL..., 1989) e fluência à compressão (FC) (INTERNATIONAL..., 2007). Os ensaios para caracterizar a capacidade de isolamento ao RI das amostras aplicadas no sistema de piso flutuante foram realizados em protótipo de câmara acústica em laboratório (Figura 3). Trata-se de uma construção em escala real, com térreo e pavimento superior, laje pré-moldada com medidas internas de $2,86 \mathrm{~m}$ por $2,42 \mathrm{~m}$, alvenaria em blocos de solo-cimento e coberta em madeiramento e telha cerâmica vermelha.

Após as medições iniciais de RI e RD, amostras de material resiliente passaram a ser submetidas à carga estática por longo prazo, com a utilização de três corpos de prova com $20 \mathrm{~N}$ cada (Figura 4). Nesse caso, utilizou-se como referência a carga correspondente ao peso próprio do contrapiso, somado às cargas acidentais estimadas para laje estrutural de dormitórios em edifícios residenciais - 1,50 kN/m segundo a NBR 6120 (ABNT, 2019).

Figura 2 - Amostras $(20 \mathrm{~cm} \times 20 \mathrm{~cm})$ dos materiais resilientes

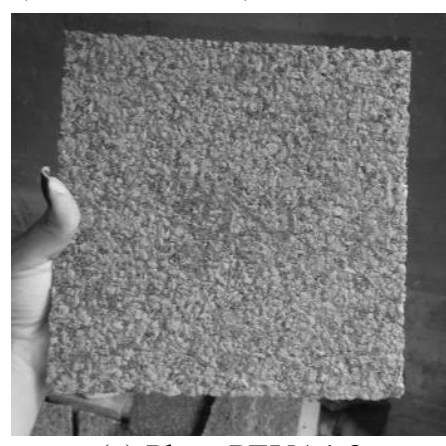

(a) Placa PEVA1,8

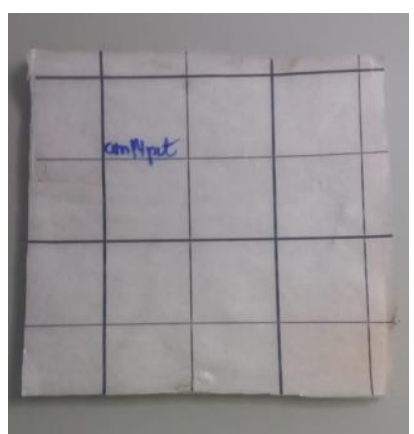

(b) Manta PET

Figura 3 - Protótipo da câmara acústica do laboratório, onde os ensaios de RI foram realizados

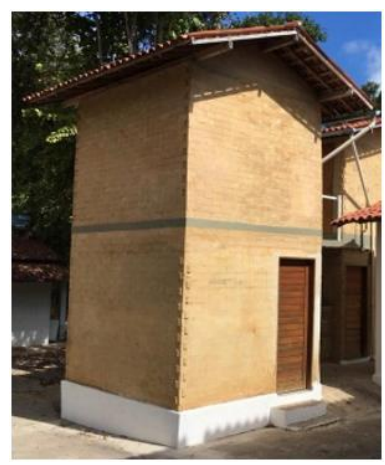

(a) Imagem

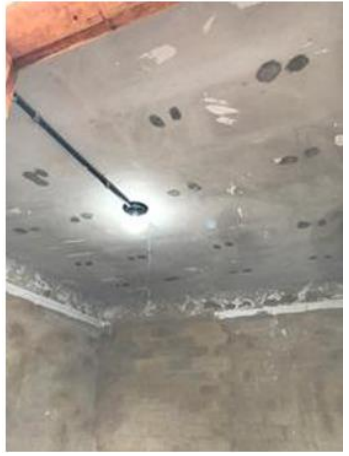

(b) Vista da laje pré-moldada na sala de recepção
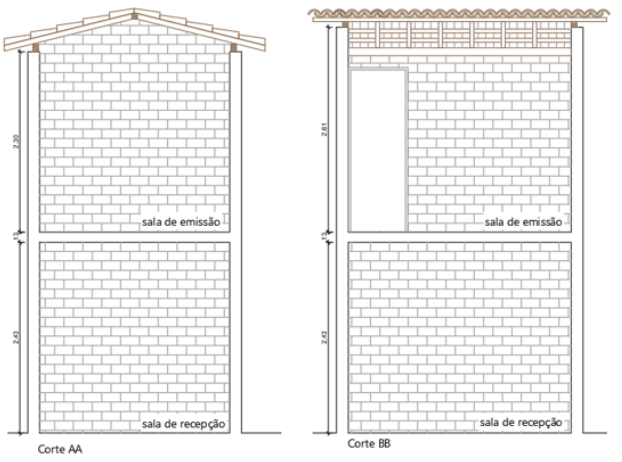

(c) Cortes esquemáticos visualizando câmaras de emissão e recepção

Figura 4 - Sistema de piso flutuante simulado em escala reduzida com os materiais resilientes submetidos à carga estática por longo prazo

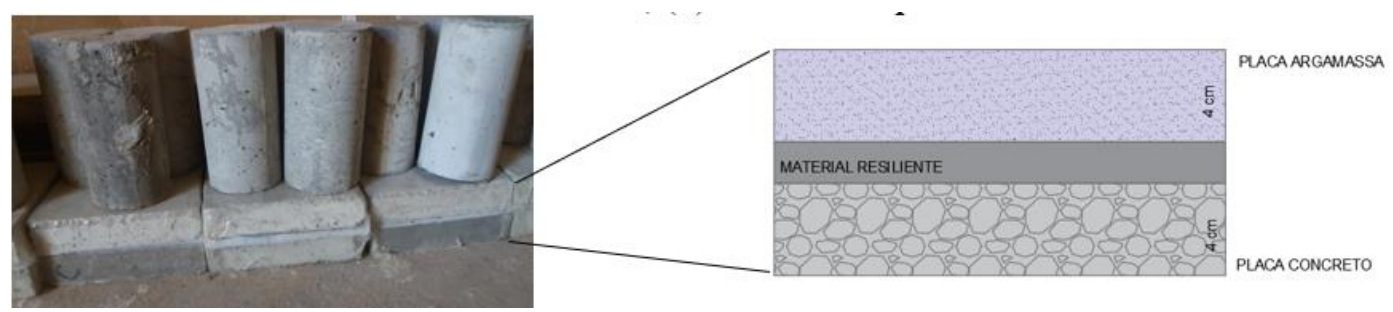

(a) Imagem parcial com as cargas estáticas sobre cada sistema

(b) Desenho esquemático do sistema 
Os períodos dessas avaliações (RI e RD) foram de até 3 anos para PEVA1,8 e de 2 anos para MantaPET, com algumas das medições repetidas a cada 12 meses. O intervalo de tempo das avaliações se deu em conformidade com o desenvolvimento da pesquisa à qual o presente estudo fez parte. O período de avaliação mais reduzido para a MantaPET foi devido à obtenção tardia de sua amostra, a qual foi doada por uma construtora após o início dos ensaios com a placa PEVA1,8. No caso das placas PEVA1,8, foram considerados dois lotes de amostras (PEVA1,8_B e PEVA1,8_C), sendo a PEVA1,8_B com as medições (RI) repetidas a cada 12 meses e o da PEVA1,8_C com tais medições somente após os $\overline{3}$ anos. Durante esses períodos todas as amostras ficaram submetidas à referida carga estática. Um terceiro lote (PEVA1,8_D) foi considerado, sem o material ser submetido à carga estática, e serviu para medição de RI inicial e para o teste de FC. Já as amostras da MantaPET tiveram dois lotes analisados, intitulados de MantaPET_A e MantaPET_B, sendo o primeiro lote com medição inicial, depois de 12 meses e ao final do segundo ano, também sub̄metido à carga estática. O segundo lote só teve análise inicial de RI, RD e análise final de FC. Quando se realizaram as medições de RI também foram feitas as medições de RD nas mesmas amostras. Os ensaios de FC das amostras PEVA1,8_D e MantaPET_B foram realizados objetivando-se simular o comportamento após 10 anos de uso. Assim como os ensaios de RD, os ensaios de FC também foram realizados em laboratório (Figura 5).

Todas as medições realizadas com os materiais resilientes utilizaram amostras em tamanho reduzido $(20 \mathrm{~cm}$ x $20 \mathrm{~cm}$ ), de modo a compatibilizar as especificidades de dimensões nos ensaios de RD e de FC. Para isso, tanto as placas PEVA1,8 (100 unidades moldadas) quanto as MantaPET foram cortadas em pedaços de 20 $\mathrm{cm} \times 20 \mathrm{~cm}$ (Figura 4).

Durante o ensaio de RI, foi montado um mosaico com amostras de cada tipo de material resiliente, para atingir $1 \mathrm{~m}^{2}$ de área. As placas ensaiadas foram justapostas abaixo de placa de argamassa já com revestimento cerâmico assentado (denominada placa flutuante), sendo esta última placa submetida aos impactos produzidos pela máquina de impactos, no interior da câmara de emissão (Figura 6).

Figura 5 - Programação dos ensaios com os materiais resilientes (PEVA1,8 e Manta PET) submetidos à carga estática

\begin{tabular}{|c|c|c|c|c|c|c|c|c|c|c|}
\hline Amostras & 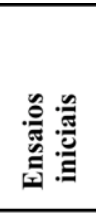 & 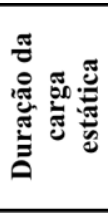 & 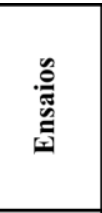 & 被 & 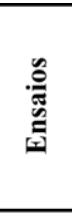 & 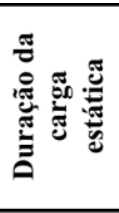 & 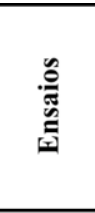 & \multirow{4}{*}{ 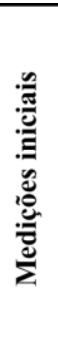 } & $\begin{array}{c}\text { Duração da } \\
\text { carga } \\
\text { estática no } \\
\text { ensaio de } \\
\text { fluência à } \\
\text { compressão }\end{array}$ & \multirow{4}{*}{ 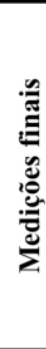 } \\
\hline PEVA1,8_B & $\mathrm{RI} / \mathrm{RD}$ & 1 ano & RI & 2 anos & RI & 3 anos & RI/RD & & \multirow{3}{*}{$\mathrm{X}$} & \\
\hline PEVA1,8_C & RI/RD & \multicolumn{5}{|c|}{3 anos } & $\mathrm{RI} / \mathrm{RD}$ & & & \\
\hline MantaPET_A & $\mathrm{RI} / \mathrm{RD}$ & 1 ano & $\mathrm{RI} / \mathrm{RD}$ & 2 anos & $\mathrm{D}$ & $\mathrm{X}$ & $\mathrm{X}$ & & & \\
\hline PEVA1,8_D & $\mathrm{RI} / \mathrm{RD}$ & \multicolumn{6}{|c|}{ sem submeter à carga estática } & $\begin{array}{l}\text { FC } \\
\text { RD }\end{array}$ & 123 dias & $\begin{array}{l}\mathrm{FC} \\
\mathrm{RD} \\
\end{array}$ \\
\hline MantaPET_B & $\mathrm{RI} / \mathrm{RD}$ & \multicolumn{6}{|c|}{ sem submeter à carga estática } & $\begin{array}{l}\mathrm{FC} \\
\mathrm{RD}\end{array}$ & 123dias & $\begin{array}{l}\mathrm{FC} \\
\mathrm{RD}\end{array}$ \\
\hline
\end{tabular}

Nota: Legenda:

$\mathrm{RI}=$ ruído de impacto;

$\mathrm{RD}=$ rigidez dinâmica; $\mathrm{e}$

FC = fluência à compressão. 
Figura 6 - Procedimentos para os ensaios de RI

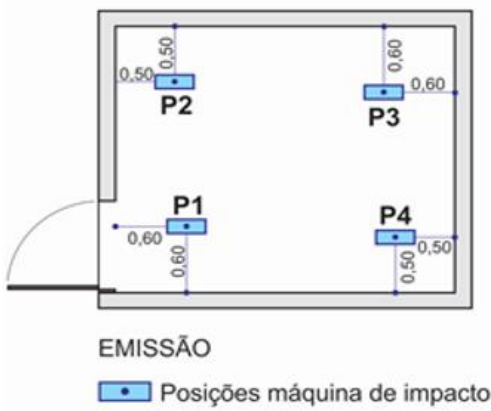

(a) Cada posição da máquina de impacto na emissãoo corresponde a quatro posições de medições com analisador acústico na recepção

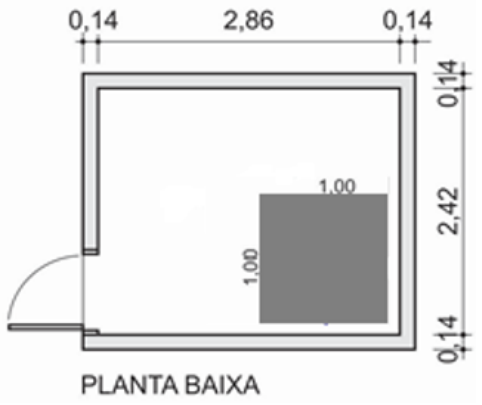

(b) Uma das quatro posições da placa flutuante

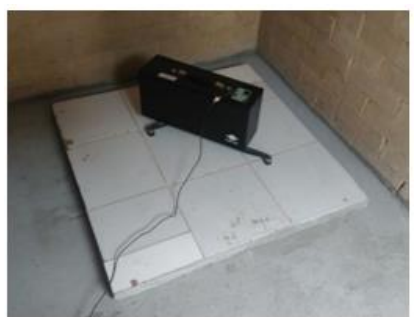

(c) Imagem da máquina de impacto sobre a placa flutuante (medidas em m)

Os testes de RI utilizando esse tipo de placa flutuante $(1 \mathrm{~m})$ tiveram como referência os estudos de Tutikian et al. (2013) e Miskinis et al. (2012), que validaram o procedimento quando se pretende comparar desempenho de materiais resilientes diferentes, desde que os testes sejam feitos com a mesma placa flutuante e sem o conjunto (placa e material resiliente) fazer contato com as paredes no perímetro do ambiente.

A sistematização dos dados coletados nos ensaios de RI resultou na determinação dos níveis de ruído de impacto padronizado $\left(\mathrm{L}_{\mathrm{nT}}\right)$, em $\mathrm{dB}$, para cada frequência, em $\mathrm{Hz}$, os quais foram aplicados em cálculos prescritos na ISO 717-2 (INTERNATIONAL..., 2013), para determinar o número único ( ${ }^{\prime}{ }_{\mathrm{nT}, \mathrm{w}}$, em $\mathrm{dB}$ ), cujo valor representa o desempenho acústico do sistema ensaiado. A letra $\mathrm{T}$ nos símbolos dos descritores $\left(\mathrm{L}{ }_{\mathrm{nT}}\right.$ e $\left.\mathrm{L}_{\mathrm{nT}, \mathrm{w}}\right)$ é utilizada para indicar resultados com correção do tempo de reverberação do ambiente de testes.

O ensaio do tempo de reverberação seguiu a NBR 11957 (ABNT, 1988), medido no mínimo em três posições de microfone, com pelo menos duas medições em cada posição do analisador acústico e uma posição da fonte sonora.

O procedimento do ensaio de RD ocorreu com a colocação do material resiliente entre duas superfícies horizontais, uma base sem irregularidades (piso do ambiente no laboratório) e uma placa metálica (placa de carga com $20 \mathrm{~cm}$ x $20 \mathrm{~cm}$ ) com massa igual a $8 \mathrm{~kg}$, conforme preconizado na ISO 9052-1 (INTERNATIONAL..., 1989). Sobre a placa metálica foram colocados acelerômetros (Figura 7a), sendo utilizado um sistema de aquisição de dados NI CompactDAQ, conectado a notebook, com o auxílio de um software específico, para visualizar os sinais captados pelos acelerômetros, a partir da excitação individual de cada amostra, realizada com martelo de borracha. Espectros em frequência dos sinais adquiridos para cada amostra ensaiada possibilitaram identificar pico correspondendo à frequência de ressonância. Com a frequência de ressonância foi possível calcular a rigidez dinâmica aparente $(s$ 't $)$ por unidade de área das amostras, utilizando a Equação 2.

Os ensaios de FC das amostras de materiais resilientes foram realizados com amostras dos lotes PEVA1,8_D e MantaPET_B, que não foram submetidas à carga estática por longo tempo. Os testes consistiram na aplicação de carga de compressão nas amostras $(20 \mathrm{~cm}$ x $20 \mathrm{~cm})$, de acordo com a ISO 20392 (INTERNATIONAL..., 2007). Foram colocadas três unidades de cada amostra, apoiadas em placas de concreto (base robusta) e sobre elas uma placa metálica com $8 \mathrm{~kg}$ de massa (Figuras 7b e 7c).

A partir da variação da espessura do material resiliente (média de quatro leituras por amostra, com uso de paquímetro digital, precisão de $0,01 \mathrm{~mm}$ ), submetido a tal compressão, durante 123 dias, estimou-se sua deformação para um período de 10 anos, calculada com base na Equação 3 e com parâmetros $m$ e $b$ de 0,11 e 0,08 respectivamente para a placa PEVA1,8_D e 0,079 e 0,26 para a MantaPET_B, obtidos nos cálculos. Na sequência, foi calculada a deformação relativa de cada amostra, $\varepsilon_{t}$, em porcentagem, estimada também para 10 anos de uso em piso flutuante, usando a Equação 4.

A estimativa do comportamento quanto ao isolamento de ruídos de impacto de cada material resiliente que estaria inserido em um piso flutuante foi determinada de acordo com a ISO 12354-2 (INTERNATIONAL..., 2017), a partir da variação do nível de pressão sonora do ruído impacto ( $\Delta \mathrm{L}$, em $\mathrm{dB})$, calculada com base na Equação 5 e utilizando a rigidez dinâmica obtida após o ensaio de FC. 
Figura 7 - Imagens dos ensaios

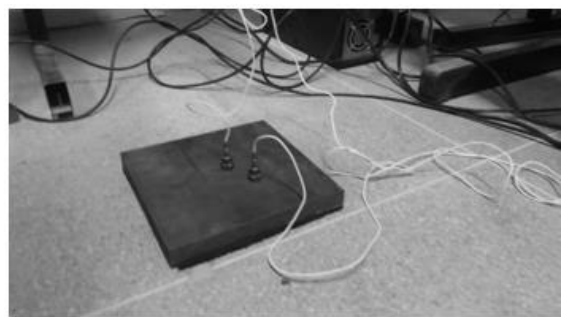

(a) $\mathrm{RD}$ com o material resiliente abaixo da placa metálica com dois acelerômetros posicionados

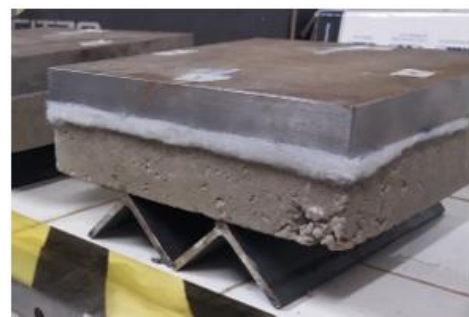

(b) FC com a placa metálica sobre a MantaPET_B

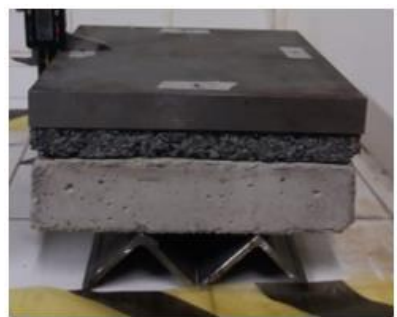

(c) FC com a placa metálica sobre a placa PEVA1,8_D

\section{Resultados e discussão}

Após a sistematização dos dados aplicando-se os valores dos níveis de ruído de impacto obtidos com ensaio seguindo a ISO 16283-2 (INTERNATIONAL..., 2015) e os respectivos tempos de reverberação, e ruído de fundo de acordo com ensaios da NBR 11957 (ABNT..., 1988), têm-se os seguintes valores para o número global ( $\mathrm{L}_{\mathrm{nT}, \mathrm{w}}$ ) das amostras, obtidos conforme a ISO 717-2 (INTERNATIONAL..., 2013) a partir das medições realizadas (vide Figura 8). A classificação do desempenho, de acordo com a NBR 15575- 3 (ABNT, 2013), está apresentada na Tabela 1.

Em relação à capacidade de isolamento ao ruído de impacto da placa PEVA1,8, o ensaio inicial (30 dias após a confecção da placa) resultou em $\mathrm{L}_{n \mathrm{n} \text {,w }}$ igual a $61 \mathrm{~dB}$, com redução de $14 \mathrm{~dB}$ (desempenho intermediário) em relação ao sistema de referência (REF) - não flutuante. $O{ }^{2}{ }_{\text {nT,w }}$ para a MantaPET, no ensaio inicial, foi igual a $55 \mathrm{~dB}$, com redução de $20 \mathrm{~dB}$ quando comparado à amostra de referência, atingindo o desempenho superior.

A Tabela 2 apresenta a variação dos valores de $\mathrm{L}_{n \mathrm{nT}, \mathrm{w}}$ ao longo do tempo, indicando que foi pouca a influência da compressão nas amostras no longo prazo (até 3 anos). Outro aspecto a destacar é que, enquanto a tendência para a placa PEVA1,8 é reduzir o valor de L' ${ }_{n T, w}$, para a MantaPET ocorre o contrário, ou seja, o primeiro reduz e o segundo aumenta $1 \mathrm{~dB}$ ao longo do tempo para o material sujeito à ação da compressão (cargas estáticas).

O comparativo entre os valores encontrados para a RD das amostras, PEVA1,8 (B e C) e MantaPET_A, em função dos respectivos tempos de ensaio, sob a ação da compressão (cargas estáticas), revela que a tendência para as placas PEVA1,8 (B e C) é diminuir a rigidez dinâmica ao longo do tempo, enquanto para a MantaPET_A é aumentar (Tabela 3). Existe uma diferença entre os valores iniciais de RD das placas PEVA1,8_B e PEVA1,8_C para a PEVA1,8_D devido ao fato de a fabricação desse lote e a realização do ensaio de rigidez dinâmica não ter sido no mesmo período; também houve uma evolução no procedimento de ensaio que possibilitou uma melhor precisão na aquisição dos resultados.

Esses resultados, ainda que expressem apenas tendências, estão coerentes com a também pequena variação observada na Tabela 2, na medida em que o aumento da RD do material resiliente corresponde a aumento do valor de $\mathrm{L}_{\mathrm{nT}, \mathrm{w}}$, o que significa redução da capacidade de isolamento ao ruído de impacto.

Os resultados de RD na comparação entre os materiais diferentes podem ser relacionados à estrutura interna destes, pois, segundo Borges (2015), os materiais com células abertas e com baixa densidade tendem a mudar a capacidade de deformabilidade ao longo do tempo. Na placa PEVA1,8 há uma resistência limitada na interface pasta de cimento/agregado de EVA, pois no processo de produção da placa PEVA1,8, propositadamente, foi interrompida a hidratação do cimento Portland após as primeiras 24 h, visando reduzir a densidade do componente. A compressão pode quebrar as frágeis ligações entre cimento e agregado, resultando em uma diminuição da rigidez do material. 
Tabela 1 - Número global e desempenho das amostras (ensaio de RI)

\begin{tabular}{l|c|c}
\hline \multicolumn{1}{c|}{ Amostras } & L' $_{\text {nT,w }}(\mathbf{d B})$ & Desempenho - NBR15575-3 (ABNT, 2013) \\
\hline Sistema de REF & 75 & Mínimo (M) \\
PEVA1,8 & 61 & Intermediário (I) \\
MantaPET & 55 & Superior (S) \\
\hline
\end{tabular}

Nota: $\mathrm{REF}=$ referência quando o teste é apenas com a placa de argamassa sobre a laje: PEVA1,8 = PEVA1,8_B, PEVA1,8_C e PEVA1,8_D; e

MANTAPET = MANTAPET_A e MANTAPET_B.

Figura 8 - Gráfico representativo dos valores de ruído de impacto padronizados em função da frequência $(\mathrm{Hz})$ para os ensaios de RI realizados

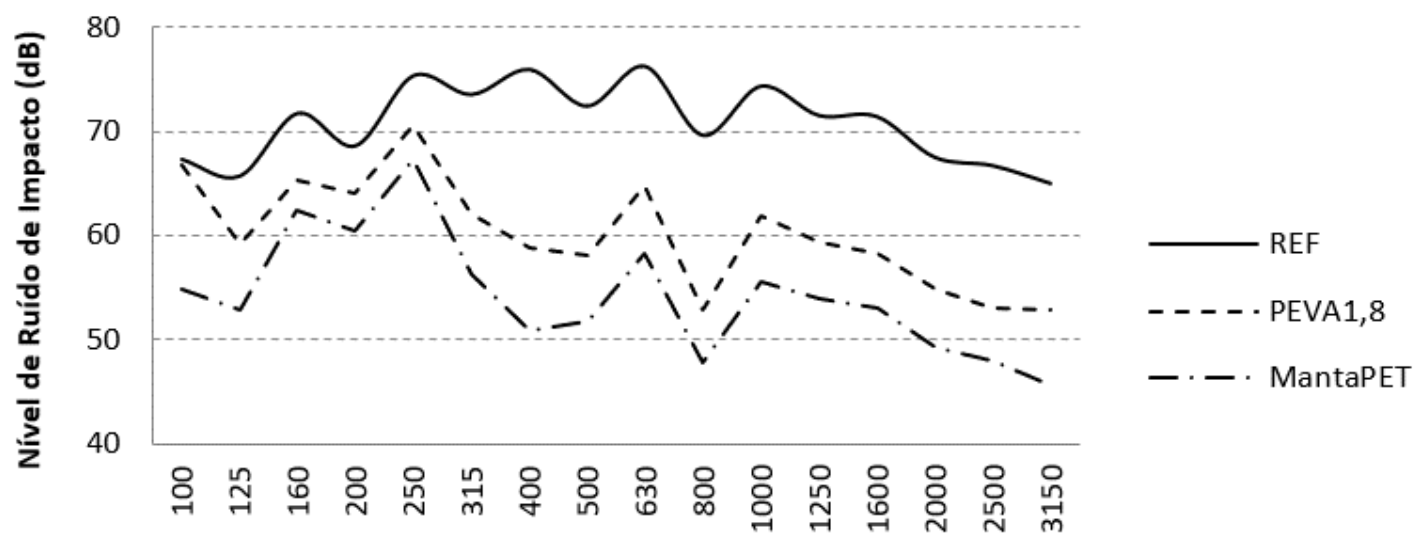

Frequências $(\mathrm{Hz})$

Tabela 2 - Valores do L' ${ }_{n T, w}$ obtidos no ensaio de RI ao longo dos 3 anos com as amostras submetidas a carga estática

\begin{tabular}{c|c|c|c|c}
\hline \multicolumn{5}{c}{ L’nT,w $^{\prime}(\mathbf{d B})$} \\
\hline Amostras & Inicial (30 dias) & Após 1 ano & Após 2 anos & Após 3 anos \\
\hline PEVA1,8_B & 61 & 62 & 59 & 60 \\
PEVA1,8_C & 61 & - & - & 60 \\
PEVA1,8_D & 61 & - & - & - \\
MantaPET_A & 55 & 56 & 56 & - \\
MantaPET_B & 55 & - & - & - \\
\hline
\end{tabular}

O mesmo não parece acontecer com a MantaPET, uma vez que é um material composto de fibras e é bastante flexível; portanto, quando é submetido a cargas estáticas de compressão, muito provavelmente suas fibras tendem a se aproximar, deixando a estrutura interna do material com menos vazios, o que pode explicar o aumento de RD. Essa análise está de acordo com Zini et al. (2016), quando afirmam que os materiais resilientes em um sistema de piso flutuante têm sua composição constantemente comprimida e que essa condição causa uma deformação, reduzindo a taxa de vazios. Nesse caso, com a redução dos vazios internos do material resiliente deve aumentar o RD, como ocorreu com a MantaPET_A. Então, uma menor deformação do material resiliente pode ser interessante, visando manter em longo prazo a propriedade de redução de ruídos de impacto, influenciada por seus vazios internos.

Com base na última medição realizada no ensaio de FC, foi possível estimar a variação na espessura de cada amostra ensaiada (Figura 9), cujo resultado para a placa PEVA1,8_ D expressa uma deformação de apenas $3,42 \%(0,73 \mathrm{~mm}$ de sua espessura inicial), sendo esse valor estimad̄o (de acordo com a Equação 3$)$ para 10 anos de uso em um sistema de piso flutuante.

A MantaPET_B apresentou uma deformação relativa de 20,15\%, obtida com a Equação 4, sendo 1,61 mm o encurtamento de sua espessura inicial $(8 \mathrm{~mm})$, obtido de acordo com a Equação 3, que é um valor estimado para 10 anos de uso em um sistema de piso flutuante. 
Tabela 3 - Comparativo entre os resultados de rigidez dinâmica das amostras em longo prazo sob ação da compressão

\begin{tabular}{c|c|c|c|c}
\hline \multicolumn{5}{c}{ Rigidez dinâmica (N/m } \\
\hline Amostras & Inicial (30 dias) & Após 1 ano & Após 2 anos & Após 3 anos \\
\hline PEVA1,8_B & 20,0 & - & - & 17,7 \\
PEVA1,8_C & 20,0 & - & - & 13,0 \\
PEVA1,8_D & 24,5 & - & - & - \\
MantaPET_A & 2,0 & 3,1 & 3,5 & - \\
MantaPET_B & 2,0 & - & - & - \\
\hline
\end{tabular}

Figura 9 - Espessuras iniciais e finais das amostras durante os ensaios de FC

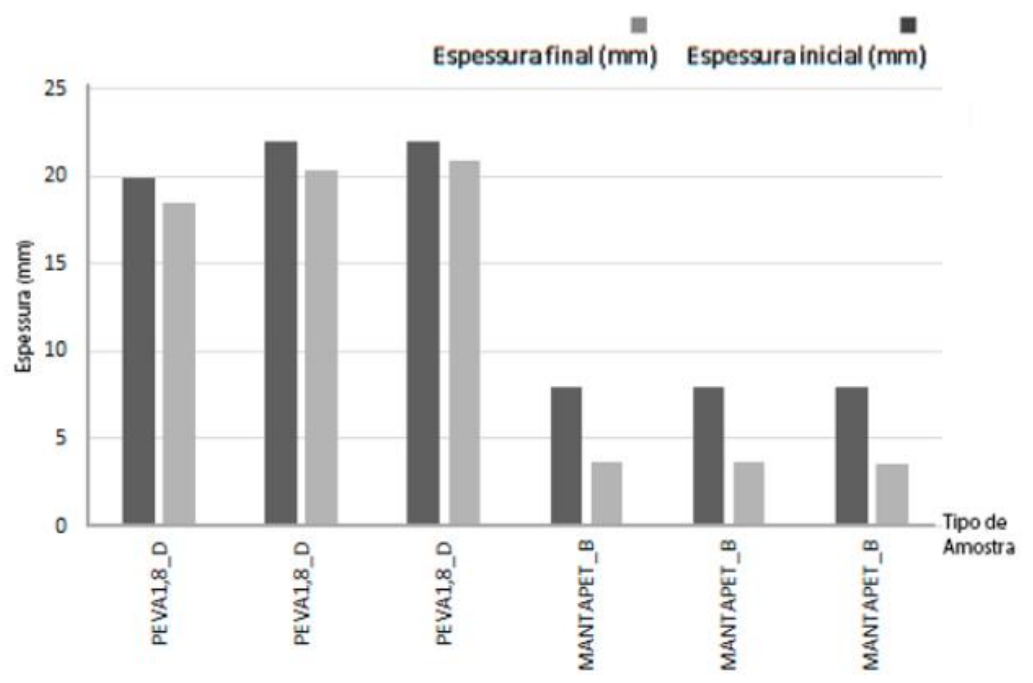

As amostras da placa PEVA1,8_D foram as que apresentaram menor deformação ao longo do tempo, seguindo a mesma tendência dos valores encontrados por Zuchetto, Nunes e Tutikyan (2015), cujo estudo com amostras compostas de argamassa com cimento Portland e EVA, com elementos com espessura inicial de $3 \mathrm{~cm}$, mostrou redução de espessura de $0,12 \mathrm{~mm}$ para o tempo estimado de 18 meses.

Borges (2015), em estudo também com amostras compostas de cimento Portland e EVA (teor de 75\%), constatou redução da espessura do componente igual a $0,17 \mathrm{~mm}$ para o mesmo período do estudo feito por Zuchetto, Nunes e Tutikyan (2015).

O estudo realizado por Kim et al. (2015) analisou a deformação no longo prazo de materiais resilientes com a presença de EVA e EPS, verificando que a espessura das amostras não mudou continuamente ao longo do tempo. Para a amostra de EVA com $2 \mathrm{~cm}$, obtiveram deformação de 13\%, e, depois de certo nível de compressão as amostras não foram mais fisicamente pressionadas. Os autores apontaram que um período de ensaio de 560 dias pode não ser suficiente para se entender completamente o fenômeno e sugeriram derivação de equação para prever uma melhor verificação da deformação no longo prazo.

O comportamento da MantaPET está de acordo com Neves, António e Nossa (2008), quando afirmam que nos materiais compressíveis a diminuição de espessura do material, quando sujeito à compressão, pode provocar o aumento de sua rigidez dinâmica. Essas observações também se apoiam nas conclusões de Klippel Filho et al. (2017), que afirmam que a deformação do material resiliente de composição fibrosa tem uma relação de dependência com sua densidade.

Após concluir os testes de FC, os cálculos feitos para a rigidez dinâmica da amostra PEVA1,8_D indicaram que o valor diminuiu 3,4\%, passando de $24,5 \mathrm{MN} / \mathrm{m}$ para 23,67 MN/m (Tabela 4). No caso da MantaPET_B, ocorreu aumento de 33,5\%, variando de 2,0 MN/m para 2,67 MN/m . 
Tabela 4 - Comparativo entre os resultados inicial e final de rigidez dinâmica das amostras após ação da compressão no ensaio de FC

\begin{tabular}{c|c|c}
\hline \multicolumn{3}{c}{ Rigidez dinâmica $\left(\mathbf{M N} / \mathbf{m}^{\mathbf{3}}\right)$} \\
\hline Amostras & Inicial & Após o teste de FC \\
\hline PEVA1,8_D & 24,5 & 23,67 \\
MantaPET_B & 2,0 & 2,67 \\
\hline
\end{tabular}

A redução do nível de pressão sonora do ruído de impacto $(\Delta \mathrm{L})$ (em relação ao piso de referência, sem manta), estimado pela rigidez dinâmica das amostras diferentes comparadas neste estudo, após os testes de FC e a partir da Equação 5, foi nula (sem variação em dB) para a placa PEVA1,8_D, sendo $\Delta \mathrm{L}$ de $28,5 \mathrm{~dB}$ na amostra antes e após a compressão, e de apenas 1,2 dB para a MantaPET_B (44,7 dB antes da compressão e 43,5 dB após).

Considerando as estimativas para 10 anos de uso dos materiais resilientes comparados e as diferenças nos valores de rigidez dinâmica após os ensaios de FC (sob carga permanente), os cálculos para $\Delta \mathrm{L}$ indicaram manutenção do nível de pressão sonora do ruído de impacto (sem variação do $\Delta \mathrm{L}$ ) para a placa PEVA1,8_D e queda de valor, ainda que de apenas 1,2 dB, para a MantaPET_B. Baseando-se em Moreira, Antonio e Tadeu (2010), esse comportamento provavelmente ocorre porque a amostra com placa PEVA1,8 contém cimento e, por isso, deforma menos e mantém o mesmo desempenho acústico ao longo do tempo. Contudo, ainda assim a MantaPET oferece melhor eficiência em termos de atenuação do ruído de impacto, comparada à placa PEVA1,8.

Com relação ao que Caniato et al. (2016) afirmam a respeito da deformação maior do que $20 \%$, como foi o caso do resultado obtido para a MantaPET_B, uma redução maior que $3 \mathrm{~dB}$ pode ser uma perspectiva para este material no longo prazo (mais que 10 anos), o que, no máximo, alteraria sua faixa de desempenho de acordo com a NBR 15575-3 (ABNT, 2013).

\section{Conclusões}

Com base nos resultados obtidos, observa-se que o procedimento adotado, tendo como base longo prazo de medições (até 3 anos) para as placas PEVA1,8_B e PEVA1,8_C, e 2 anos para MantaPET_A, possibilitou verificar a influência da compressão no desempenho acústico dos materiais resilientes. As verificações focaram na expectativa de alteração da rigidez dinâmica dos dois diferentes materiais resilientes testados (PEVA1,8 - alternativo; MantaPET - comercial), bem como na possível variação de suas capacidades de atenuação do ruído de impacto nos sistemas de pisos flutuantes simulados. Os seguintes pontos conclusivos podem ser destacados:

(a) a placa PEVA1,8_B (material resiliente alternativo) apresentou redução (até 11,5\%) na rigidez dinâmica, alterando de $20,0 \mathrm{MN} / \mathrm{m}$ ao trigésimo dia para $17,7 \mathrm{MN} / \mathrm{m}$ após 3 anos sendo submetida a compressão, mas isso não representou ganho significativo (apenas $1 \mathrm{~dB},+1,8 \%$ ) na capacidade de isolamento ao ruído de impacto no piso ( $\mathrm{L}^{\prime}{ }_{\mathrm{nT}, \mathrm{w}}$ igual a $\left.60 \mathrm{~dB}\right)$;

(b) a MantaPET_A (material resiliente comercial), ao contrário, apresentou aumento (até 75\%) na rigidez dinâmica $(2,0 \mathrm{MN} / \mathrm{m} / 3,5 \mathrm{MN} / \mathrm{m}$ ) após 2 anos submetida a compressão, mas isso também não representou perda expressiva (apenas $1 \mathrm{~dB} ;-1,6 \%$ ) na capacidade de isolamento ao ruído de impacto no piso ( $\mathrm{L}_{\mathrm{nT}, \mathrm{w}}$ igual a $56 \mathrm{~dB})$; e

(c) o teste de fluência à compressão teve como resultado uma deformação menor para a placa PEVA1,8_D (3,42\%), comparada à MantaPET_B (20,15\%), e confirmou a mesma tendência da variação da rigidez dinâmica entre os materiais, sem comprometimento do desempenho acústico deles, quando aplicados em piso flutuante. Logo, ambas as análises confirmam que, embora haja variação da rigidez dinâmica dos materiais testados, não é esperado haver alteração significativa em seus desempenhos quanto ao isolamento ao ruído de impacto depois de longo prazo instalados em sistemas de pisos flutuantes.

A placa PEVA1,8 se mostrou com potencial para ser utilizada como material resiliente alternativo em sistemas de pisos flutuantes, uma vez que a presença dos agregados leves de EVA (resíduos da indústria de calçados) no compósito cimentício proposto confere uma interessante característica ao produto devido à baixa deformação das placas ao longo do tempo, o que pode propiciar maior estabilidade como material resiliente para suportar o peso dos materiais adicionados sobre elas após a execução do piso flutuante.

136 Santos, F. A. dos; Melo, A. B. de; Pimentel, R. L.; Oliveira, M. F. de 


\section{Referências}

ASSOCIAÇÃO BRASILEIRA DE NORMAS TÉCNICAS. NBR 11957: reverberação: análise do tempo de reverberação em auditórios: método de ensaio. Rio de Janeiro, 1988.

ASSOCIAÇÃO BRASILEIRA DE NORMAS TÉCNICAS. NBR 15575-3: edifícios habitacionais de até cinco pavimentos: desempenho: parte 3: requisitos para os sistemas de pisos internos. Rio de Janeiro, 2013

ASSOCIAÇÃO BRASILEIRA DE NORMAS TÉCNICAS. NBR 6120: ações para o cálculo de estruturas de edificações. Rio de Janeiro, 2019.

BISTAFA, S. R. Acústica aplicada ao controle do ruído. São Paulo: Edgard Blucher, 2011.

BORGES, J. G. K. Análise das propriedades acústicas de contrapisos produzidos com materiais reciclados. São Leopoldo, 2015. Dissertação (Mestrado em Engenharia Civil) - Universidade do Vale do Rio dos Sinos, São Leopoldo, 2015.

BORGES, J. K. et al. An experimental study on the use of waste aggregate for acoustic attenuation: EVA and rice husk composites for impact noise reduction. Construction and Building Materials. v. 161, p. 501508, 2018.

BRANCHER, L. R. et al. Acoustic behavior of subfloor lightweight mortars containing micronized poly (ethylene vinyl acetate) EVA. Materials, v. 9, n. 51, p. 1-9, 2016.

CÂMARA BRASILEIRA PARA A INDUSTRIA DA CONSTRUÇÃO. Desempenho das edificações habitacionais: guia orientativo para atendimento à Norma 15575/2013. Brasília, 2013.

CANIATO, M. et al. Time-depending performance of resilient layers under floating floors. Construction and Building Materials, v. 102, p. 226-232, 2016.

FERRAZ, R. Atenuação de ruído de impacto em pisos de edificações de pavimentos múltiplos. Belo Horizonte, 2008. Dissertação (Mestrado em Engenharia Civil) - Universidade Federal de Minas Gerais, Belo Horizonte, 2008.

GARLET, G. Aproveitamento de resíduos de E.V.A. (ethylene vinyl acetate) como agregado para concreto leve na construção civil. Porto Alegre, 1998. Dissertação (Mestrado em Engenharia Civil) Escola de Engenharia, Universidade Federal do Rio Grande do Sul, Porto Alegre, 1998.

INTERNATIONAL ORGANIZATION FOR STANDARDIZATION. ISO 12354-2: building acoustics: estimation of acoustic performance of buildings from the performance of elements: part 2: impact sound insulation between rooms. Geneve, 2017.

INTERNATIONAL ORGANIZATION FOR STANDARDIZATION. ISO 16283-2: acoustic: field measurement of sound insulation in buildings and of building elements: part 2: Impact sound insulation. Geneve, 2015.

INTERNATIONAL ORGANIZATION FOR STANDARDIZATION. ISO 20392: thermal-insulating materials: determination of compressive creep. Geneve, 2007.

INTERNATIONAL ORGANIZATION FOR STANDARDIZATION. ISO 717-2: acoustic: rating of sound insulation in building elements: part 2: impact sound insulation. Geneve, 2013.

INTERNATIONAL ORGANIZATION FOR STANDARDIZATION. ISO 9052-1: acoustics: determination of dynamic stiffness: part 1: materials used under floating floors in dwellings. Geneve, 1989.

KIM, K. et al. Influence of loading time of a load plate and sample size on the measurement of physical properties of resilient materials. Archives of Acoustics, v. 40, n. 2, p. 159-167, 2015.

KLIPPEL FILHO, S. et al. Uso da lã de PET para a absorção sonora e o isolamento acústico. Acústica e Vibrações, v. 49, p. 59-69, 2017.

MISKINIS, K. et al. Dependence between reduction of weighted impact sound pressure level and specimen size of floating floor construction. Material Science, Medziagotyra, v. 18, n. 1, 2012.

MOREIRA, A.; ANTONIO, J.; TADEU, A. Impact sound reduction of concrete layers containing cork granules. In: INTER-NOISE CONGRESS \& EXPOSITION ON NOISE CONTROL ENGINEERIN, 39. Lisboa, 2010. Proceedings [...] Lisboa, 2010. 
NEVES, A.; ANTÓNIO, J.; NOSSA, A. Resultados experimentais da rigidez dinâmica de materiais usados sob pavimentos flutuantes. Coimbra, 2008. Disponível em: http://www.seaacustica.es/fileadmin/Coimbra08/id212.pdf. Acesso em: 12 jan. 2020.

NUNES, M. F. O.; ZINI, A.; PAGNUSSAT, D. T. Desempenho acústico de sistemas de piso: estudos de caso para isolamento ao ruído aéreo e de impacto. Acústica e Vibrações, v. 46, p. 13-19, 2014.

SCHIAVI, A. et al. Acoustical and mechanical characterization of an innovative expanded sintered elasticized polystirene (EPS-E) used as underlayer in floating floors. In: INTERNATIONAL CONGRESS ON ACOUSTICS, 19., Madrid, 2007. Proceedings [...] Madrid, 2007.

SOUZA, L. C. L.; ALMEIDA, M. G.; BRAGAN, A. L. Bê-á-bá da acústica arquitetônica: ouvindo a arquitetura. São Carlos: EdUFSCar, 2006.

TUTIKIAN, B. F. et al. Lightweight concrete with EVA recycled aggregate for impact noise attenuation. Materiales de Construcción, v. 63, n. 310, p. 309-316, 2013.

TUTIKIAN, B. F. et al. Uso de agregado leve de EVA em contrapiso argamassado para isolamento ao ruído de impacto em edificações residenciais. Ambiente Construído, Porto Alegre, v. 17, n. 3, p. 295-306, jul./set. 2017.

ZINI, A. et al. Sound insulation of floors: a new composite with a resilient layer of recycled polymer. Journal of Civil Engineering and Architecture, v. 10, p. 861-869, 2016.

ZUCHETTO, L. K.; NUNES, M. F. O.; TUTIKYAN, B. F. Dynamic stiffness evaluation of floor covering system made out of recycled EVA: ethylene vinyl acetate. In: INTER-NOISE CONGRESS \& EXPOSITION ON NOISE CONTROL ENGINEERING, 44., São Francisco, 2015. Proceedings [...] São Francisco, 2015.

Fabianne Azevedo dos Santos

Programa de Pós-Graduação em Engenharia Civil e Ambiental | Universidade Federal da Paraíba | Via Expressa Padre Zé, s/n, Centro de Tecnologia, Campus I, Castelo Branco | João Pessoa - PB - Brasil | CEP 58051-900 | Tel: (83) 3216-7393 | E-mail:

fabianne_as@hotmail.com

\section{Aluísio Braz de Melo}

Departamento de Arquitetura e Urbanismo | Universidade Federal da Paraíba | Cidade Universitária, Campus I João Pessoa - PB - Brasil | CEP 58051-900 | Tel: (83) 3216-7200 | E-mail: aluisiobmelo@hotmail.com

Roberto Leal Pimentel

Departamento de Engenharia Civil | Universidade Federal da Paraíba | Cidade Universitária | João Pessoa - PB - Brasil | CEP 58051-900 | Tel: (83) 32167711 | E-mail: r.pimentel@uol.com.br

Maria Fernanda de Oliveira

itt Performance | Universidade do Vale dos Sinos | Av. Unisinos, 950, Cristo Rei | São Leopoldo - RS - Brasil | CEP 93022-750 | Tel: (51) 3590-8887 | E-mail: mariaon@unisinos.br

Ambiente Construído

Revista da Associação Nacional de Tecnologia do Ambiente Construído

Av. Osvaldo Aranha, $99-3^{\circ}$ andar, Centro

Porto Alegre - RS - Brasil CEP $90035-190$

Telefone: +55 (51) 3308-4084

www.seer.ufrgs.br/ambienteconstruido www.scielo.br/ac

E-mail: ambienteconstruido@ufrgs.br 